\title{
Patient-Reported Experience and Outcome Measures in People Living with Diabetes: A Scoping Review of Instruments
}

\author{
Jimmy Martin-Delgado ${ }^{1,2,3} \cdot$ Mercedes Guilabert $^{4} \oplus$ - José Mira-Solves ${ }^{1,4,5} \oplus$
}

Accepted: 16 May 2021 / Published online: 27 May 2021

(c) The Author(s) 2021

\begin{abstract}
Background Diabetes mellitus is a global public health concern, with over 463 million people living with this chronic disease. Pathology complexity, management difficulty, and limited participation in care has resulted in healthcare systems seeking new strategies to engage people living with diabetes. Patient-reported experience measures (PREMs) and patient-reported outcome measures (PROMs) were developed to address the gap between the healthcare system expectation and patient preference.

Objective This study aimed to review the existing literature on PREMs and PROMs specific to type 1 and 2 diabetes, and report the dimensions report the dimensions they have measured.

Methods A scoping review was conducted from January 1985 to March 2020 of six databases, MEDLINE, EMBASE, PsycINFO, CINHAL, Scopus, and BiblioPro, to identify PREM and PROM instruments specific for type 1 and 2 diabetes. Results Overall, 34 instruments were identified, 32 PROMs and two PREMs. The most common instrument included outcomes related to quality of life at $44 \%(n=15)$, followed by satisfaction (whether with treatment, device, and healthy habits) at 26\% $(n=9)$. Furthermore, instruments regarding personal well-being accounted for $15 \%(n=5)$. For instruments that measure experiences of persons with diabetes, there were two scales of symptoms, and one related to the attitude patients have toward the disease.

Conclusions Diabetes-specific validated instruments mainly focus on quality of life, education, and treatment, and sometimes overlap each other, in their subscales and assessment dimensions. Constructs such as cultural and religious beliefs, leisure, and work life may need more attention. There appears to be a gap in instruments to measure experiences of individuals who "live with diabetes" and seek to lead a "normal life."
\end{abstract}

Jimmy Martin-Delgado

jimmy.martind@umh.es

1 Atenea Research Group, Foundation for the Promotion of Health and Biomedical Research, Health District Alicante-Sant Joan, Carretera Nacional 332, Av. de Benidorm, Sant Joan d'Alacant, 03550 Alicante, Spain

2 Health Services and Policy Research Group, University of Exeter Medical School, Exeter, UK

3 Department of Public Health, Universidad Católica de Santiago de Guayaquil, Guayaquil, Ecuador

4 Department of Health Psychology, Miguel Hernández University, Elche, Spain

5 Research Network on Health Services in Chronic Diseases (REDISSEC), Alicante, Spain

\section{Key Points for Decision Makers}

Disease-specific instruments are tailored to patient needs, avoiding irrelevancies from other generic measures.

It is vital to use an adequate instrument, with an adequate construct for measurement.

Patient-reported outcome measure instruments usually focus on patients' quality of life; other aspects such as the social context should also be considered.

We found no cross-culturally validated or on-site developed instruments to be used in low-income and middleincome countries; more research is needed. 


\section{Introduction}

Chronic non-communicable diseases pose a challenge for healthcare systems. The global prevalence of diabetes mellitus among adults (aged 20-79 years) was estimated at 9.3\% (463 million people) in 2019, and is expected to rise to $10.2 \%$ (578 million) by 2030 [1]. Patient-centered care can be challenging to define, but common concepts are: disease and illness experience (patient-as-person), whole person (biopsychosocial perspective), common ground (sharing power and responsibility with healthcare provider), and patient-doctor relationship [2].

Since the 1990s, research has increasingly placed quality of life (QoL) in the spotlight, as an essential health outcome in diabetes, in some cases, if not "the ultimate goal" of treatment [3]. Currently, clinical trials should include the evaluation of the psychological aspects of patients, treatment satisfaction, well-being, and QoL, which are referred to as patient-reported outcome measures (PROMs) [4, 5]. For better intervention on healthdisease processes, a patient with a chronic condition must be an active protagonist $[6,7]$. Therefore, it is essential to incorporate the patient's viewpoints into the organization of healthcare. Accordingly, it has been verified that when the patient perceives that his/her perspective, experience, and decisions about the illness are taken into account, there is more active and effective participation and cooperation in the treatment plan $[8,9]$. The incorporation of instruments known as patient-reported experience measures (PREMs) has made it possible to make the patient visible within the context of the healthcare systems and include their experience in the management of chronic pathologies. The PREM and PROM instruments respond to the objectives of achieving patient-centered care.

Diabetes prevention and control are challenges for healthcare and social professionals, services, and systems. It also has a high economic and social impact [10]. The promotion of healthy lifestyles, the control of risk factors (diet, weight management, physical exercise), diabetes education, and patient self-care are essential elements in controlling the disease's progression and the social and health burden it represents [11]. However, these are not the only factors that need to be considered. Significant evidence exists regarding the influence of psychosocial stress on risk factors, acceleration of disease, and overall health of individuals [12]. A recent study showed that daily stress related to work and an increased perceived risk of physical health may influence outcomes in diabetes care [13].

The experiences and results measured by patients change according to the local socio-cultural context. Healthcare systems and their results are not homogeneous and could change within the same region. Accordingly, it is necessary to understand how patients construct and interpret their disease. Healthcare systems need to adapt to patients' needs, create a climate of trust, and act to improve adherence and the quality of care [14].

This study aimed to review disease-specific patientreported outcomes and experience measures that currently exist for individuals with type 1 and 2 diabetes and what dimensions they measure. We attempt to provide a synthesis of these instruments for policy makers and researchers in order for them to choose the most suitable instrument for their intended purpose.

\section{Methods}

A scoping review of studies published in peer-reviewed journals was conducted. The Joanna Briggs Institute [15] protocol has been followed, and the Preferred Reporting Items for Systematic Reviews and Meta-Analyses-Extension for Scoping Reviews (PRISMA-ScR) checklist was used [16]. The study protocol is available from the corresponding author.

\subsection{Inclusion Criteria}

Studies specific to type 1 and 2 diabetes that collected, developed, or validated PROMs were included to examine: (a) QoL, (b) emotional stress, (c) symptoms, (d) adherence to treatment, and (e) satisfaction with treatment; and experience measures such as (1) living with diabetes and (2) the healthcare system. As there were no restrictions on age, the studies were selected from the young, adult, and older populations. Studies in both English and Spanish were included. Articles validating instruments on other diseases such as gestational diabetes, diabetic foot ulcers, selection of nutritional supplements, and other non-specific diseases such as high blood pressure, metabolic syndrome, or cardiovascular disease were excluded.

\subsection{Data Sources and Search Strategy}

The selected databases included MEDLINE, EMBASE, PsycINFO (via OvidSP), CINHAL, Scopus, and BiblioPro. The research team agreed on the terms and appropriately modified each database. The articles were limited to the English and Spanish languages, published between January 1985 and March 2020. Specific publication types were excluded from the search strategy, such as editorials and case reports. A combination of Medical Subject Headings $(\mathrm{MeSH})$ and free-text terms were used. Three groups of terms were generated to describe: (1) the population; (2) instruments, surveys, and PREMs/PROMs; and (3) psychometric properties. Terms within each group were combined 
with the Boolean operator "OR." BiblioPro is a patientreported measures database available in Spanish, and it was manually searched to look for possible instruments. In a complementary method, manual searches were performed in Google Scholar to identify the gray literature in different countries, which are not available in the already-mentioned scientific databases. The OvidSP database search is available in the Electronic Supplementary Material.

\subsection{Selection of Reviewers}

JMD conducted the search strategy in the databases. JMD and MG reviewed all titles and abstracts to identify potentially relevant studies. When a consensus was not achieved between both reviewers, a third researcher (JJM) reviewed the study and disagreements were addressed to reach a consensus. All the reviewers could read Spanish and English. Studies in the local language included an abstract that allowed the authors to decide if it had to be included for full-text reading. JMD and MG performed full-text reading of articles for inclusion. No unpublished studies were found, and neither was there a need to contact any of the authors included.

\subsection{Data Extraction}

Data extraction from each study was performed by all authors using a standardized Excel sheet. The sheet included the year of publication, whether PROM or PREM, number of items, outcome, dimensions, target population, mode of administration, recall period, number of participants, response options, range of scores, administration time, original language, and number of citations. Information about the design and the validation procedure used in each instrument was also included.

\subsection{Data Synthesis}

A qualitative and descriptive analysis of the data was conducted for each variable from which information was extracted. The researchers generated categories according to dimensions and its most recent definitions. The presence or absence of information in each variable was coded. Heterogeneity in the methods and measures applied was described when possible.

\subsection{Quality Evaluation}

The Consensus-based Standards for the Selection of Health Measurement Instruments (COSMIN) checklist was employed to evaluate the studies' methodological rigor [17]. The psychometric results reported in the studies were described and categorized into the COSMIN measurement properties.

\section{Results}

Overall, 8220 records were identified, of which 6003 remained after the exclusion of duplicates. After the review of titles and abstracts, 5783 studies were eliminated. Consequently, 220 articles were assessed with fulltext reading, of which 193 were excluded, of these 173 were not diabetes-specific instruments, 15 did not include any patient-reported measure, four were related to ulcer foot care, and one was a nutritional scale. After the inclusion of two articles identified by searches of references, 29 articles meeting all inclusion criteria were included. Figure 1 shows this process of selecting studies.

\subsection{Description of the Studies}

Patient-reported outcome measure instruments were the most prevalent, accounting for $94 \%(n=32)$ and PREM instruments, only $6 \%(n=2)$. In the last 10 years, a notable increase was observed with the instruments, thus doubling the number. Regarding the instruments, outcomes related to QoL were the most common at $44 \%(n=15)$, followed by those that measured satisfaction (whether with treatment, device, or healthy habits) at $26 \%(n=9)$, and the well-being of the person accounted at $15 \%(n=5)$. Two scales of symptoms associated with diabetes and one instrument related to the attitude of patients having the disease complete the selected outcome measures. Finally, only two instruments that measure the experiences of the person with diabetes were found. The number of items showed a high variability range from 7 to 41 . Of these instruments, 13 were specific for application in type 2 diabetes, unlike 17 instruments used indistinctly in the two most common presentations of pathology (types 1 and 2), thereby leaving only four specific instruments for patients with type 1 diabetes. Most of the instruments were designed for the adult population, representing the $85 \%$ $(n=29)$. Table 1 shows the main characteristics of the selected instruments.

English was the predominant language for the development of the selected instruments, accounting for $74 \%$ ( $n$ $=25$ ). The most cited PROM is the Summary of Diabetes Self Care [18], and the sample size is heterogenous, with a range from 146 to 3594 participants in the included studies. Table 2 includes information related to the mode of administration, recall period, number of participants, 
Fig. 1 Preferred Reporting Items for Systematic Reviews and Meta-Analyses-Extension for Scoping Reviews (PRISMA$\mathrm{ScR}$ ) diagram. PREM patientreported experience measure, PROM patient-reported outcome measure
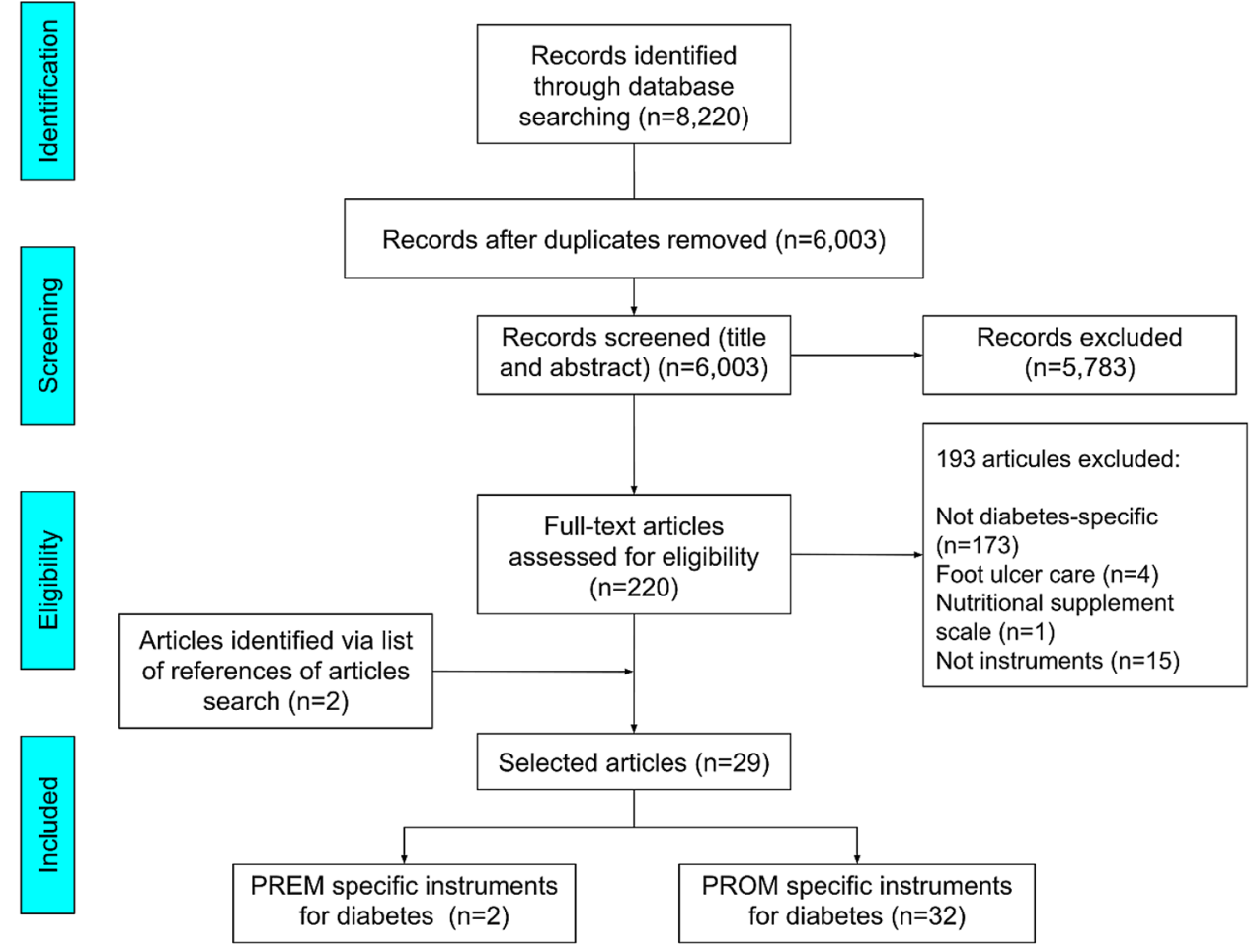

response options, range of scores, administration time, original language, and the number of citations.

\subsection{Dimensions}

The selected instruments independently reported a total of 75 dimensions. Different constructs were included such as emotional distress, where the most commonly used instrument is Problem Areas in Diabetes (PAID) [19]. Healthy lifestyles and habits such as the Diabetes Health Profile 1 [20] and 18 [21], or the Diabetes Self-Management Questionnaire (DSMQ) [22]; patient education and engagement, where the Summary of Diabetes Self Care [18] and more recently the Diabetes Intention, Attitude and Behavior Questionnaire (DIAB-Q) [23] are conspicuous. Others were related to mobility, self-sufficiency in daily activities, treatment, emotional support (of the social circle or caregiver), and hypoglycemia or hyperglycemia symptoms. However, instruments specific to patients with type 1 diabetes tend to include questions related to satisfaction with the medical device, such as the Treatment Related Impact Measure for Diabetes Device (TRIM-DD) [24] or expectations about insulin treatment, and Expectations About Insulin Therapy (EAITQ) [25]. The other dimensions measured but in PREM instruments were patients' perceived experience concerning healthcare, communication with the professional, care planning, care delivery, and patient-centered care. Table 3 includes a summary of all dimensions of selected instruments after review of instrument composition.

\subsection{Quality Evaluation}

The most frequently reported property was construct validity $(n=29)$, followed by content and criterion validity $(n=28)$, face validity $(n=20)$, and responsiveness $(n=17)$. Internal consistency was usually presented as Cronbach's $\alpha(n=25)$ with values of over $>0.70$. In 16 studies, Cronbach's $\alpha$ was presented as a single sum of the total scale and its dimensions, as opposed to nine studies that presented values for each of the dimensions. Test-retest was the least reported psychometric quality, only 15 studies (44\%), and in most of the cases, authors reported the result of the intra-class correlation coefficient. Table 4 includes properties of each of the selected studies.

\section{Discussion}

Undoubtedly, QoL and patients' experiences and feelings should be assessed using qualitative methods, but we also need quantitative measures for clinical trials or for evaluating healthcare services [8, 47]. Outcome measures based on patient feedback are indispensable to move toward 


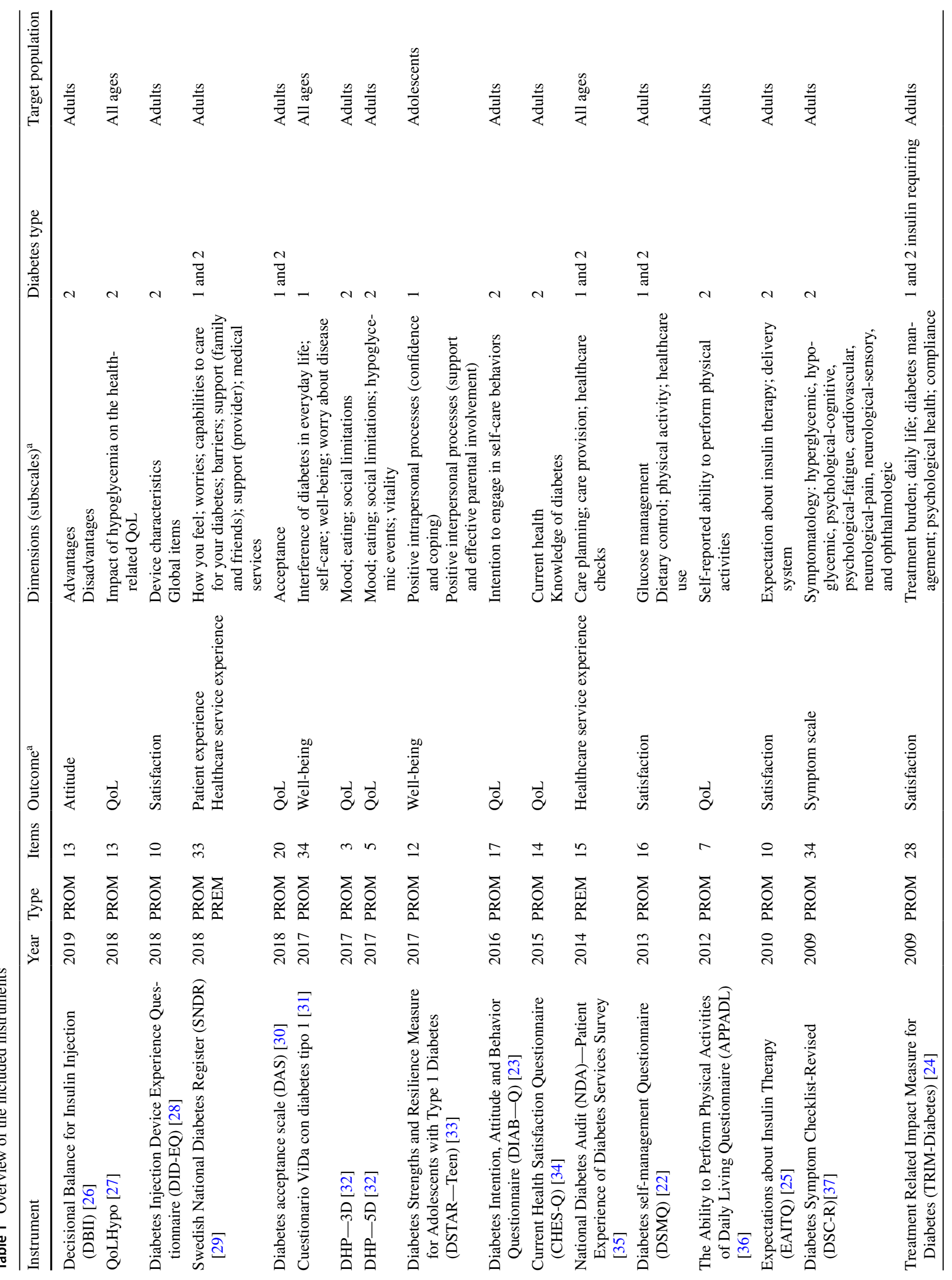




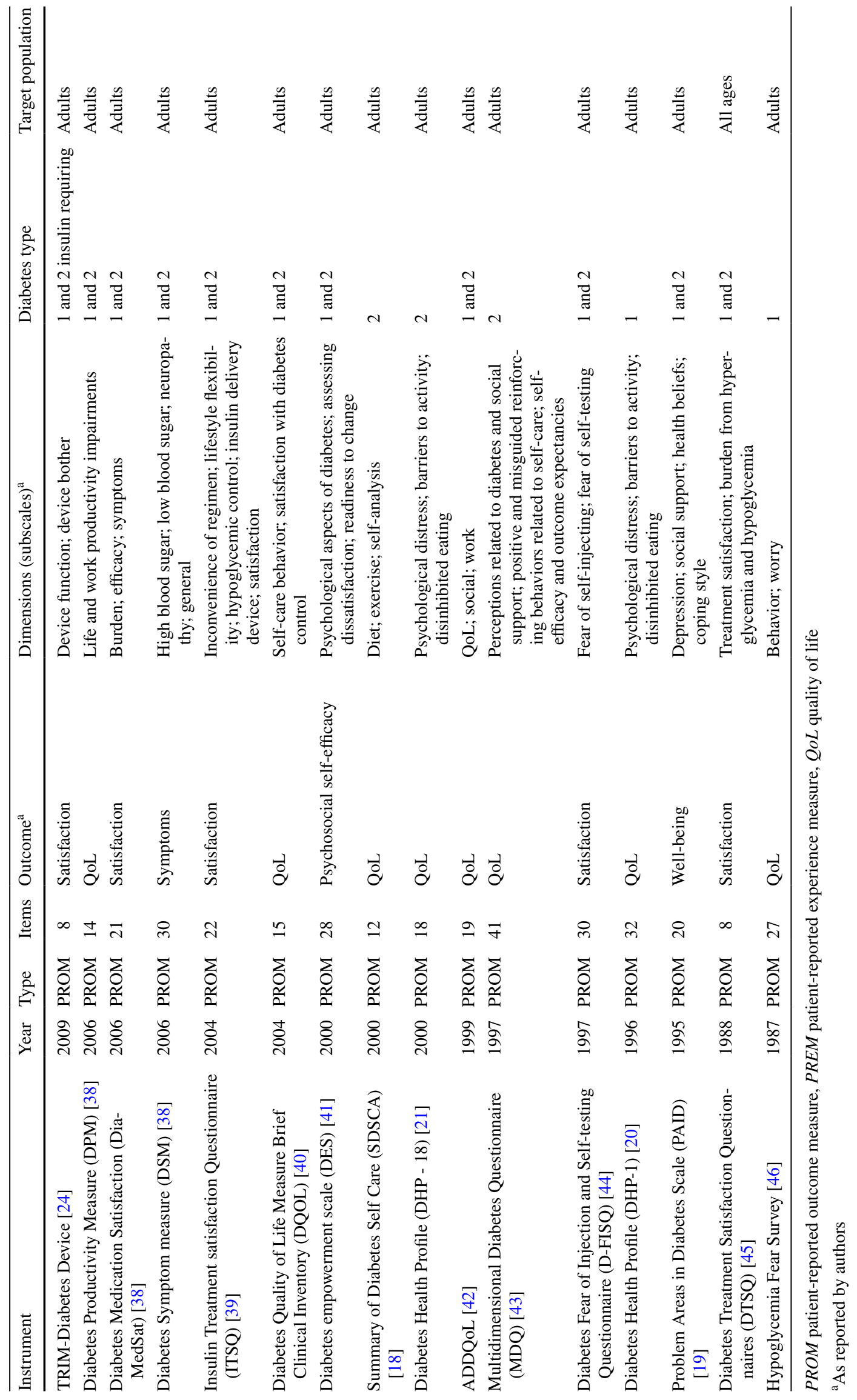


Table 2 Selected studies characteristics

\begin{tabular}{|c|c|c|c|c|c|c|c|c|}
\hline Instrument & $\begin{array}{l}\text { Mode of } \\
\text { administration }\end{array}$ & Recall period & Participants & $\begin{array}{l}\text { Response } \\
\text { options }\end{array}$ & $\begin{array}{l}\text { Range of } \\
\text { scores }\end{array}$ & $\begin{array}{l}\text { Administra- } \\
\text { tion time, } \\
\text { minutes }\end{array}$ & $\begin{array}{l}\text { Original } \\
\text { language }\end{array}$ & $\begin{array}{l}\text { Num- } \\
\text { ber of } \\
\text { citations }^{a}\end{array}$ \\
\hline DBII [26] & - & - & 332 & $\begin{array}{l}\text { Strongly } \\
\text { disagree/ } \\
\text { strongly } \\
\text { agree }\end{array}$ & $1-5$ & - & Taiwanese & 2 \\
\hline QoLHypo [27] & Paper sheet & - & 227 & Never/always & $0-2$ & - & Spanish & 2 \\
\hline DID-EQ [28] & Mail & $\begin{array}{l}\text { Present per- } \\
\text { ception }\end{array}$ & 142 & $\begin{array}{l}\text { Strongly } \\
\text { disagree/ } \\
\text { strongly } \\
\text { agree } \\
\text { Not at all } \\
\text { confident/ } \\
\text { completely } \\
\text { confident } \\
\text { Very dissatis- } \\
\text { fied/very } \\
\text { satisfied } \\
\text { Very difficult/ } \\
\text { very easy }\end{array}$ & $1-4$ & - & English & 5 \\
\hline SNDR [29] & Mail & - & 972 & - & - & - & Swedish & 14 \\
\hline DAS [30] & Paper sheet & 2 weeks & 606 & $\begin{array}{l}\text { Never true for } \\
\text { me/always } \\
\text { true for me }\end{array}$ & $0-3$ & - & German & 14 \\
\hline $\begin{array}{c}\text { Cuestionario } \\
\text { ViDa [31] }\end{array}$ & Paper sheet & - & 578 & $\begin{array}{l}\text { Strongly } \\
\text { disagree/ } \\
\text { strongly } \\
\text { agree }\end{array}$ & $1-5$ & 30 & Spanish & 7 \\
\hline DHP - 3D [32] & Interview & - & 150 & $\begin{array}{l}\text { Never/always } \\
\text { Very easy/not } \\
\text { at all easy }\end{array}$ & $0-3$ & - & English & 4 \\
\hline $\begin{array}{c}\text { DHP-- 5D } \\
{[32]}\end{array}$ & Interview & - & 150 & $\begin{array}{l}\text { Never/always } \\
\text { Very easy/not } \\
\text { at all easy } \\
\text { None of the } \\
\text { time/all of } \\
\text { the time }\end{array}$ & $0-3$ & - & English & 4 \\
\hline $\begin{array}{l}\text { DSTAR-Teen } \\
\text { [33] }\end{array}$ & Online & - & 260 & $\begin{array}{l}\text { Never/almost } \\
\text { always }\end{array}$ & $1-5$ & - & English & 28 \\
\hline DIAB - Q [23] & Online & 1 week & 1015 & $\begin{array}{l}\text { Strongly } \\
\text { disagree/ } \\
\text { strongly } \\
\text { agree } \\
\text { Not at all } \\
\text { valuable/ } \\
\text { extremely } \\
\text { valuable }\end{array}$ & $1-7$ & - & English & 10 \\
\hline CHES-Q [34] & Online & $\begin{array}{l}\text { Present per- } \\
\text { ception }\end{array}$ & 1015 & $\begin{array}{l}\text { Strongly } \\
\text { disagree/ } \\
\text { strongly } \\
\text { agree }\end{array}$ & $1-7$ & - & English & 10 \\
\hline NDA [35] & Online & 12 months & 714 & - & - & - & English & 21 \\
\hline DSMQ [22] & - & 8 weeks & 261 & $\begin{array}{l}\text { Does not } \\
\text { apply to } \\
\text { me/applies } \\
\text { to me very } \\
\text { much }\end{array}$ & $0-3$ & - & German & 269 \\
\hline
\end{tabular}


Table 2 (continued)

\begin{tabular}{|c|c|c|c|c|c|c|c|c|}
\hline Instrument & $\begin{array}{l}\text { Mode of } \\
\text { administration }\end{array}$ & Recall period & Participants & $\begin{array}{l}\text { Response } \\
\text { options }\end{array}$ & $\begin{array}{l}\text { Range of } \\
\text { scores }\end{array}$ & $\begin{array}{l}\text { Administra- } \\
\text { tion time, } \\
\text { minutes }\end{array}$ & $\begin{array}{l}\text { Original } \\
\text { language }\end{array}$ & $\begin{array}{l}\text { Num- } \\
\text { ber of } \\
\text { citations }^{\mathrm{a}}\end{array}$ \\
\hline APPADL [36] & Paper sheet & $\begin{array}{l}\text { Present per- } \\
\text { ception }\end{array}$ & 146 & $\begin{array}{l}\text { Unable to do/ } \\
\text { not at all } \\
\text { difficult }\end{array}$ & $1-5$ & $<5$ & English & 9 \\
\hline EAITQ [25] & Paper sheet & - & 240 & $\begin{array}{l}\text { Strongly } \\
\text { disagree/ } \\
\text { strongly } \\
\text { agree }\end{array}$ & $1-7$ & - & English & 9 \\
\hline DSC-R [37] & Paper sheet & 4 weeks & 3594 & $\begin{array}{l}\text { Not at all/ } \\
\text { extremely }\end{array}$ & $1-5$ & - & English & 73 \\
\hline TRIM-D [24] & Online & 2 weeks & 507 & $\begin{array}{l}\text { Not at all } \\
\text { satisfied/ } \\
\text { extremely } \\
\text { satisfied } \\
\text { Not at all } \\
\text { convenient/ } \\
\text { extremely } \\
\text { convenient } \\
\text { Never/almost } \\
\text { never Inter- } \\
\text { feres/almost } \\
\text { Always/ } \\
\text { always } \\
\text { interferes }\end{array}$ & $1-5$ & 5 & English & 65 \\
\hline $\begin{array}{l}\text { TRIM-DD } \\
\text { [24] }\end{array}$ & Online & 2 weeks & 507 & - & $1-5$ & 1 & English & 65 \\
\hline DPM [38] & Online & - & 409 & - & - & $<2$ & English & 88 \\
\hline $\begin{array}{l}\text { Dia-MedSat } \\
\text { [38] }\end{array}$ & Online & - & 409 & - & - & 4 & English & 88 \\
\hline DSM [38] & Online & - & 409 & - & - & 2 & English & 88 \\
\hline ITSQ [39] & Online & 4 weeks & 402 & $\begin{array}{l}\text { No bother at } \\
\text { all/a tremen- } \\
\text { dous bother } \\
\text { Does not } \\
\text { interfere at } \\
\text { all/interferes } \\
\text { tremen- } \\
\text { dously } \\
\text { No planning } \\
\text { at all/a } \\
\text { tremendous } \\
\text { amount of } \\
\text { planning } \\
\text { Extremely } \\
\text { confident/ } \\
\text { not at all } \\
\text { confident } \\
\text { Not at all } \\
\text { worried/ } \\
\text { extremely } \\
\text { worried }\end{array}$ & $1-7$ & - & English & 112 \\
\hline $\begin{array}{l}\text { Brief DQOL } \\
\quad[40]\end{array}$ & Mail & - & 498 & $\begin{array}{l}\text { Never/all the } \\
\text { time } \\
\text { Very satisfied/ } \\
\text { very dis- } \\
\text { satisfied }\end{array}$ & $1-5$ & - & English & 150 \\
\hline
\end{tabular}


Table 2 (continued)

\begin{tabular}{|c|c|c|c|c|c|c|c|c|}
\hline Instrument & $\begin{array}{l}\text { Mode of } \\
\text { administration }\end{array}$ & Recall period & Participants & $\begin{array}{l}\text { Response } \\
\text { options }\end{array}$ & $\begin{array}{l}\text { Range of } \\
\text { scores }\end{array}$ & $\begin{array}{l}\text { Administra- } \\
\text { tion time, } \\
\text { minutes }\end{array}$ & $\begin{array}{l}\text { Original } \\
\text { language }\end{array}$ & $\begin{array}{l}\text { Num- } \\
\text { ber of } \\
\text { citations }^{\mathrm{a}}\end{array}$ \\
\hline DES [41] & Mail & - & 375 & $\begin{array}{l}\text { Strongly } \\
\text { agree/ } \\
\text { strongly } \\
\text { disagree }\end{array}$ & $1-5$ & - & English & 693 \\
\hline SDSCA [18] & Mail & 7 days & 127 & $\begin{array}{c}\text { How many } \\
\text { days have } \\
\text { you ... }\end{array}$ & $0-7$ & - & English & 2400 \\
\hline DHP - 18 [21] & Mail & - & 426 & $\begin{array}{l}\text { Never/always } \\
\text { Never/very } \\
\text { often } \\
\text { Not at all/very } \\
\text { much } \\
\text { Very likely/ } \\
\text { not at all } \\
\text { likely }\end{array}$ & $0-3$ & 7 & English & 97 \\
\hline ADDQoL [42] & Paper sheet & - & 154 & $\begin{array}{l}\text { A great deal } \\
\text { better/a } \\
\text { great deal } \\
\text { worse } \\
\text { Increased a } \\
\text { great deal/ } \\
\text { decreased a } \\
\text { great deal }\end{array}$ & -3 to +3 & - & English & 678 \\
\hline MDQ [43] & Mail & - & 249 & $\begin{array}{l}\text { Not at all } \\
\text { important/ } \\
\text { very impor- } \\
\text { tant }\end{array}$ & $\begin{array}{l}0-6 \\
0-100\end{array}$ & - & French & 225 \\
\hline D-FISQ [44] & Mail & Past month & 266 & Never/always & $0-3$ & - & Dutch & 67 \\
\hline DHP-1 [20] & Mail & - & 2239 & $\begin{array}{l}\text { Never/always } \\
\text { Never/very } \\
\text { often } \\
\text { Not at all/very } \\
\text { much } \\
\text { Very likely/ } \\
\text { not at all } \\
\text { likely }\end{array}$ & $0-3$ & - & English & 150 \\
\hline PAID [19] & Paper sheet & - & 451 & $\begin{array}{l}\text { No problem/ } \\
\text { serious } \\
\text { problem }\end{array}$ & $0-6$ & - & English & 1251 \\
\hline DTSQ [45] & Paper sheet & Few weeks & 219 & $\begin{array}{l}\text { Very dissatis- } \\
\text { fied/very } \\
\text { satisfied } \\
\text { None of the } \\
\text { time/most of } \\
\text { the time } \\
\text { Very flexible/ } \\
\text { very inflex- } \\
\text { ible }\end{array}$ & $0-6$ & - & English & 420 \\
\hline $\begin{array}{l}\text { Hypoglycemia } \\
\text { Fear Survey } \\
\text { [46] }\end{array}$ & Paper sheet & - & 158 & $\begin{array}{l}\text { Never/very } \\
\text { often }\end{array}$ & $1-5$ & - & English & 539 \\
\hline
\end{tabular}

Please refer to Table 1 for the full instrument name

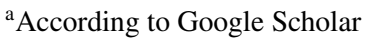


Table 3 Dimensions reported in the selected instruments

\begin{tabular}{|c|c|c|c|c|c|c|c|c|c|}
\hline \multirow[t]{2}{*}{ Instrument } & \multicolumn{9}{|c|}{ Dimensions } \\
\hline & QoL & $\begin{array}{l}\text { Emotional } \\
\text { distress }\end{array}$ & Lifestyle & $\begin{array}{l}\text { Education and } \\
\text { engagement }\end{array}$ & Treatment $^{\mathrm{a}}$ & Support & Social & $\begin{array}{l}\text { Medical } \\
\text { services }\end{array}$ & Symptoms \\
\hline DBII [26] & & & & & $\checkmark$ & & & & \\
\hline QoLHypo [27] & $\checkmark$ & & & & & & & & \\
\hline DID-EQ [28] & & & & & $\checkmark$ & & & & \\
\hline SNDR [29] & & $\checkmark$ & & $\checkmark$ & & $\checkmark$ & $\checkmark$ & $\checkmark^{b}$ & \\
\hline DAS [30] & & $\checkmark$ & $\checkmark$ & & & & & & \\
\hline Cuestionario ViDa [31] & $\checkmark$ & $\checkmark$ & $\checkmark$ & $\checkmark$ & & & & & \\
\hline DHP-3D [32] & & $\checkmark$ & $\checkmark$ & & & & & & \\
\hline DHP-5D [32] & $\checkmark$ & $\checkmark$ & $\checkmark$ & & & & & & \\
\hline DSTAR-Teen [33] & & & & $\checkmark$ & & $\checkmark$ & & & \\
\hline $\mathrm{DIAB}-\mathrm{Q}[23]$ & & & & $\checkmark$ & & & & & \\
\hline CHES-Q [34] & $\checkmark$ & & & $\checkmark$ & & & & & \\
\hline NDA [35] & & & & & & & & $\checkmark^{b}$ & \\
\hline DSMQ [22] & & & $\checkmark$ & & $\checkmark$ & & & & \\
\hline APPADL [36] & & & $\checkmark$ & & & & & & \\
\hline EAITQ [25] & & & & & $\checkmark$ & & & & \\
\hline DSC-R [37] & & & & & & & & & $\checkmark$ \\
\hline TRIM-D [24] & & $\checkmark$ & $\checkmark$ & & $\checkmark$ & & & & \\
\hline TRIM-DD [24] & & & & & $\checkmark$ & & & & \\
\hline DPM [38] & & & $\checkmark$ & & & & & & \\
\hline Dia-MedSat [38] & & & & & $\checkmark$ & & & & $\checkmark$ \\
\hline DSM [38] & & & & & & & & & $\checkmark$ \\
\hline ITSQ [39] & & & $\checkmark$ & & $\checkmark$ & & & & \\
\hline Brief DQOL [40] & & & & $\checkmark$ & $\checkmark$ & & & & \\
\hline DES [41] & & $\checkmark$ & & $\checkmark$ & $\checkmark$ & & & & \\
\hline SDSCA [18] & & & $\checkmark$ & $\checkmark$ & & & & & \\
\hline $\mathrm{DHP}-18[21]$ & $\checkmark$ & $\checkmark$ & $\checkmark$ & & & & & & \\
\hline ADDQoL[42] & $\checkmark$ & & & & & $\checkmark$ & $\checkmark$ & & \\
\hline MDQ [43] & & & $\checkmark$ & $\checkmark$ & & & $\checkmark$ & & \\
\hline D-FISQ [44] & & & & & $\checkmark$ & & & & \\
\hline DHP-1 [20] & $\checkmark$ & $\checkmark$ & $\checkmark$ & & & & & & \\
\hline PAID [19] & & $\checkmark$ & $\checkmark$ & $\checkmark$ & & $\checkmark$ & & & \\
\hline DTSQ [45] & & $\checkmark$ & & & $\checkmark$ & & & & \\
\hline Hypoglycemia Fear Survey [46] & & $\checkmark$ & & $\checkmark$ & & & & & \\
\hline
\end{tabular}

Please refer to Table 1 for the full instrument name

QoL quality of life

${ }^{a}$ Treatment satisfaction or dissatisfaction, satisfaction with device, satisfaction with insulin treatment, fear of self-testing

${ }^{\mathrm{b}}$ Stands for patient-reported experience measure dimensions; care planning, care provision, healthcare checks, and provider support

person-centered care. These metrics should cover all dimensions relevant to them. Patients with diabetes are often under significant psychological distress because of strict adherence to medications, changes in their daily activities, patterns such as diet and exercise, and fear of long-term macrovascular and microvascular complications, which will undermine their QoL [48].

Diabetes-related distress is defined as patients' concerns about the self-management of diabetes, perception of support, emotional burden, and access to quality healthcare [49], and is associated with poorer glycemic control and QoL [50-52]. This life experience of the individual with diabetes needs to be measured to provide strategies to cope with diabetes-related distress and properly achieve patientcentered care [53]. However, the traditional management and decisions made in diabetes have focused on reducing glycated hemoglobin levels for the prevention of long-term complications. This approach can incur in treatments that are 
Table 4 Evaluation of psychometric properties

\begin{tabular}{|c|c|c|c|c|c|c|c|}
\hline \multirow[t]{2}{*}{ Instrument } & \multicolumn{2}{|l|}{ Reliability } & \multicolumn{3}{|l|}{ Validity } & \multirow[b]{2}{*}{$\begin{array}{l}\text { Construct } \\
\text { validity }\end{array}$} & \multirow{2}{*}{$\begin{array}{l}\text { Respon } \\
\text { sivenes }\end{array}$} \\
\hline & Internal consistency & Test-retest & Face validity & $\begin{array}{l}\text { Content } \\
\text { validity }\end{array}$ & $\begin{array}{l}\text { Criterion } \\
\text { validity }\end{array}$ & & \\
\hline DBII [26] & $\alpha=0.78-0.92$ & - & - & $\checkmark$ & - & $\checkmark$ & - \\
\hline QoLHypo [27] & $\alpha=0.901$ & $\mathrm{ICC}=0.92$ & $\checkmark$ & $\checkmark$ & $\checkmark$ & $\checkmark$ & $\checkmark$ \\
\hline DID-EQ [28] & $\alpha=0.80$ & $\mathrm{ICC}=0.92$ & - & $\checkmark$ & $\checkmark$ & $\checkmark$ & $\checkmark$ \\
\hline SNDR [29] & - & Weighted Kappa $=0.78$ & $\checkmark$ & $\checkmark$ & - & - & $\checkmark$ \\
\hline DAS [30] & $\alpha=0.96$ & $r=0.89$ & $\checkmark$ & $\checkmark$ & $\checkmark$ & $\checkmark$ & $\checkmark$ \\
\hline Cuestionario ViDa [31] & $\alpha=0.71-0.86$ & $r=0.78$ & $\checkmark$ & $\checkmark$ & $\checkmark$ & $\checkmark$ & $\checkmark$ \\
\hline $\mathrm{DHP}-3 \mathrm{D}[32]$ & - & - & - & - & - & - & - \\
\hline $\mathrm{DHP}-5 \mathrm{D}[32]$ & - & - & - & - & - & - & - \\
\hline DSTAR-Teen [33] & $\alpha=0.89$ & - & $\checkmark$ & $\checkmark$ & $\checkmark$ & $\checkmark$ & - \\
\hline $\mathrm{DIAB}-\mathrm{Q}[23]$ & $\alpha=0.30-0.68$ & $\mathrm{ICC}=0.63-0.84$ & $\checkmark$ & $\checkmark$ & $\checkmark$ & $\checkmark$ & $\checkmark$ \\
\hline CHES-Q [34] & - & $\mathrm{ICC}=0.63-0.83$ & $\checkmark$ & $\checkmark$ & $\checkmark$ & $\checkmark$ & $\checkmark$ \\
\hline NDA [35] & - & - & - & - & - & - & - \\
\hline DSMQ [22] & $\alpha=0.84$ & - & $\checkmark$ & $\checkmark$ & $\checkmark$ & $\checkmark$ & - \\
\hline APPADL [36] & $\alpha=0.89$ & $\mathrm{ICC}=0.91$ & - & $\checkmark$ & $\checkmark$ & $\checkmark$ & $\checkmark$ \\
\hline EAITQ [25] & $\alpha=0.82$ & $\mathrm{ICC}=0.80$ & $\checkmark$ & $\checkmark$ & $\checkmark$ & $\checkmark$ & - \\
\hline DSC-R [37] & $\alpha=0.69-0.87$ & - & $\checkmark$ & $\checkmark$ & $\checkmark$ & $\checkmark$ & $\checkmark$ \\
\hline TRIM-D [24] & $\alpha=0.94$ & $\mathrm{ICC}=0.85$ & $\checkmark$ & $\checkmark$ & $\checkmark$ & $\checkmark$ & $\checkmark$ \\
\hline TRIM-DD [24] & $\alpha=0.80$ & $\mathrm{ICC}=0.89$ & $\checkmark$ & $\checkmark$ & $\checkmark$ & $\checkmark$ & $\checkmark$ \\
\hline DPM [38] & $\alpha=0.95$ & - & $\checkmark$ & $\checkmark$ & $\begin{array}{l}\checkmark \\
\checkmark\end{array}$ & $\checkmark$ & - \\
\hline Dia-MedSat [38] & $\alpha=0.79-0.91$ & - & $\checkmark$ & $\checkmark$ & $\checkmark$ & $\checkmark$ & - \\
\hline DSM [38] & $\alpha=0.95$ & - & $\checkmark$ & $\checkmark$ & $\checkmark$ & $\checkmark$ & - \\
\hline ITSQ [39] & $\alpha=0.92$ & $r=0.94$ & $\checkmark$ & $\checkmark$ & $\checkmark$ & $\checkmark$ & $\checkmark$ \\
\hline Brief DQOL [40] & $\alpha=0.85$ & - & - & $\checkmark$ & $\checkmark$ & $\checkmark$ & - \\
\hline DES [41] & $\alpha=0.96$ & $\mathrm{ICC}=0.79$ & - & - & $\checkmark$ & $\checkmark$ & $\checkmark$ \\
\hline SDSCA [18] & $\mathrm{IIC}=0.20-0.77$ & $\mathrm{ICC}=0.53$ & $\checkmark$ & $\checkmark$ & $\checkmark$ & $\checkmark$ & $\checkmark$ \\
\hline $\mathrm{DHP}-18[21]$ & $\alpha=0.71-0.88$ & - & - & $\checkmark$ & $\checkmark$ & $\checkmark$ & - \\
\hline ADDQoL [42] & $\alpha=0.85$ & - & $\checkmark$ & $\checkmark$ & $\checkmark$ & $\checkmark$ & - \\
\hline MDQ [43] & $\alpha=0.70-0.91$ & - & - & $\checkmark$ & $\checkmark$ & $\checkmark$ & - \\
\hline D-FISQ [44] & $\alpha=0.94$ & - & - & - & $\checkmark$ & $\checkmark$ & - \\
\hline DHP-1 [20] & $\alpha=0.77-0.86$ & - & $\checkmark$ & $\checkmark$ & $\checkmark$ & $\checkmark$ & $\checkmark$ \\
\hline PAID [19] & $\alpha=0.95$ & - & - & $\checkmark$ & $\checkmark$ & $\checkmark$ & - \\
\hline DTSQ [45] & $\alpha=0.76$ & - & $\checkmark$ & $\checkmark$ & $\checkmark$ & $\checkmark$ & $\checkmark$ \\
\hline Hypoglycemia Fear Survey [46] & $\alpha=0.90$ & $\mathrm{ICC}=0.89$ & - & $\checkmark$ & $\checkmark$ & $\checkmark$ & $\checkmark$ \\
\hline
\end{tabular}

Please refer to Table 1 for the full instrument name

$\alpha$ Cronbach $\alpha$ coefficient, ICC intra-class correlation coefficient, $r$ Spearman rank correlation coefficient

complex and intrusive with the patient's QoL. In the last two decades, attempts have been made to measure the relationship between glycemic control and psychosocial functioning [9, 54, 55].

The last disease-specific, health-related QoL systematic review for diabetes was conducted more than a decade ago [56]. Since then, research on PROMs has gained ground, doubling in number as shown in the present study. In this review, only specific diabetes instruments have been considered because these are tailored to meet patients' needs. Generic instruments assess constructs or feelings relevant for anyone, leaving aside domains such as lifestyle flexibility or interference of diabetes in everyday life [57].

The correct use of the most appropriate instruments must be accurately determined by the researcher, focusing on the outcome pursued in the protocol [58]. Frequently, instruments are not appropriately selected for the purpose as explained by Speight et al. [3]. Additionally, we should 
also consider that dimensions can overlap. If we group the reported dimensions of the selected studies according to what they actually ask and measure, the characteristics would be as follows: lifestyle represents $20 \%(n=14)$, treatment (includes satisfaction with treatment and devices) represents $18 \%(n=13)$, emotional distress represents $17 \%$ $(n=12)$, education represents $15 \%(n=11)$, QoL represents $10 \%(n=7)$, social relations and support represent $13 \%(n=9)$, and finally, others (includes general questions and symptoms) represent $4 \%(n=3)$. Conversely, PREM instruments address patients' perception of medical services, and the following dimensions were reported, care planning, care provision, healthcare checks, and support provided by healthcare professionals.

Among the most widely used PROMs for measuring the QoL of patients with diabetes are the ADDQoL, the WHO Well-Being questionnaire, and the EuroQoL-5D. However, two of these three are not specific to diabetes [3]. The three most cited scales are SDSCA (2400), PAID (1251), and DES (693). If we include ADDQoL, they mainly focus on healthy lifestyles, diabetes-related distress, and social support. Aspects such as whether individuals received integrated health and social care when required, how their occupational or leisure activities with friends and family are affected, or how their social and religious customs are respected within the framework of the healthcare they receive are areas that have not received sufficient attention in these instruments, although these are relevant issues for patients [59].

Research has predominantly focused on type 2 diabetes; in this review, only one PROM was specifically designed for adolescents with type 1 diabetes [33]. The psychometric properties of the included studies are reported in most of the instruments, and the administration time varies widely from 2 to $30 \mathrm{~min}$. Nevertheless, this may not be enough, as $60 \%$ of them are paper based, and even though half of them have been developed in the last 10 years, online application has not taken over, and they do not seem to be systematically implemented. Barriers for proper implementation of PROMs have been identified, including time in the implementation process, lack of access and support in the inner setting, preparing an organization for implementation, especially training clinicians, and the inability of patients to complete instruments in the intervention process $[60,61]$.

Not all of the included studies considered patients during the development process of the instrument. To achieve person-centered care, individuals need to be part of the healthcare system and process. Qualitative studies have shown that the personal life experience of "living with diabetes" may be influenced by many barriers including social, economic, and cultural beliefs, personal factors, education, access to information, provider support, and medical treatment $[62,63]$.

Chronicity, defined as the combination of effects and complications that non-communicable diseases have on patients, is a challenge for all healthcare systems worldwide. Evaluation frameworks such as Triple AIM [64] or the Chronic Care Model [65] propose a shift in the measurement of outcomes and call for new metrics that incorporate health and social intervention experience and lifetime outcomes. The PREM and PROM instruments, such as those included in this review, have been widely used to assess these chronicity strategies implemented by healthcare services $[35,66$, 67]. For people with diabetes, it is particularly relevant to address chronicity because of the comorbidity and impact of the disease on their QoL. These instruments can be used in both population-based and experimental studies focused on measuring an intervention's effectiveness from the patient's point of view. However, some of them, because of their length, may be impractical and have a more research-focused approach.

Undoubtedly, there are many instruments, developed in different countries by various healthcare systems or researchers, that mostly focus on patient satisfaction with their treatment, QoL, or fear of complications from treatment. Of these instruments, $78 \%$ were designed in the USA or the UK and were in English, the remainder in Spain (Spanish), Sweden (Swedish), Canada (French), the Netherlands (Dutch), and Germany (German). All of them were developed in high-income countries with strong economies and robust healthcare systems. Disease-specific measures are tailored to patient needs; it is not the same construct or feeling between different chronic diseases. To avoid irrelevancies that could cloud the picture, disease-specific measures will have a greater sensitivity. Generic measures (depressed mood, anxiety, energy, and positive well-being) may be common to everybody, but they will not remain constant across different conditions. Hyperglycemic and hypoglycemic episodes or chronic complications may disrupt these concepts and are confounded in people with diabetes [68]. Therefore, there might be a current need to develop diabetes-specific instruments in low-income and middle-income countries, where social, cultural and economic contexts and access to healthcare are different [69].

There were certain limitations in conducting this review. Despite diabetes being one of the most prevalent chronic non-communicable diseases, there is limited information about pathology-specific PREM instruments, unlike PROMs that have been widely developed over the past few years. This study only included variables related to diabetes, thus any other instrument that has not been specifically developed for patients with diabetes has not been included in the review process. 


\section{Conclusions}

It is imperative to harmonize healthcare and direct our efforts in knowing the life experience of a patient in the face of his/her pathology. While there are specific validated instruments for people with diabetes, many of them overlap each other, in their subscales and assessment dimensions. The most developed instrument accounts for those related to education, QoL, and satisfaction, leaving the door open for the development of new instruments that measure the experience of a person who "lives with diabetes" and seeks to lead a "normal life."

Supplementary Information The online version contains supplementary material available at https://doi.org/10.1007/s40271-021-00526-y.

Acknowledgements We thank Dr. Jose M. Valderas for his valuable input into this study. Jimmy Martin-Delgado is supported by a Santiago Grisolia Scholarship (GrisoliaP/2018/158).

\section{Declarations}

Funding This study was supported by Prometeu/2017/173 grant (Generalitat Valenciana, 2017).

Conflicts of Interest/Competing Interests Jimmy Martin-Delgado, Mercedes Guilabert, and José Mira-Solves have no conflicts of interest that are directly relevant to the content of this article.

Ethics Approval This study is part of a wider project with approval of the Ethics Committee of the Kennedy Clinical Hospital of Guayaquil (HCK-CEISH-19-0041).

Consent to Participate Not applicable.

Consent for Publication Not applicable.

Availability of Data and Material Data and material are available from the corresponding author upon reasonable request.

Code Availability Not applicable.

Author's Contributions All authors contributed to the study conception and design. JM performed the literature search and database equations. JM and MG double checked the literature search results. Data analysis was performed by all the authors. The first draft was written by JM and MG. All authors critically revised the work and approved its final version for publication.

Open Access This article is licensed under a Creative Commons Attribution-NonCommercial 4.0 International License, which permits any non-commercial use, sharing, adaptation, distribution and reproduction in any medium or format, as long as you give appropriate credit to the original author(s) and the source, provide a link to the Creative Commons licence, and indicate if changes were made. The images or other third party material in this article are included in the article's Creative Commons licence, unless indicated otherwise in a credit line to the material. If material is not included in the article's Creative Commons licence and your intended use is not permitted by statutory regulation or exceeds the permitted use, you will need to obtain permission directly from the copyright holder. To view a copy of this licence, visit http://creativecommons.org/licenses/by-nc/4.0/.

\section{References}

1. Saeedi P, Petersohn I, Salpea P, Malanda B, Karuranga S, Unwin $\mathrm{N}$, et al. Global and regional diabetes prevalence estimates for 2019 and projections for 2030 and 2045: results from the International Diabetes Federation Diabetes Atlas, 9th edition. Diabetes Res Clin Pract. 2019;157:107843.

2. Hudon C, Fortin M, Haggerty JL, Lambert M, Poitras M-E. Measuring patients' perceptions of patient-centered care: a systematic review of tools for family medicine. Ann Fam Med. 2011;9:155-64.

3. Speight J, Reaney MD, Barnard KD. Not all roads lead to Rome: a review of quality of life measurement in adults with diabetes. Diabet Med. 2009;26:315-27.

4. Valderas JM, Alonso J. Patient reported outcome measures: a model-based classification system for research and clinical practice. Qual Life Res. 2008;17:1125-35.

5. Deshpande P, Sudeepthi BL, Rajan S, Abdul NC. Patientreported outcomes: a new era in clinical research. Perspect Clin Res. 2011;2:137-44.

6. Kinmonth AL, Spiegal N, Woodcock A. Developing a training programme in patient-centred consulting for evaluation in a randomised controlled trial; diabetes care from diagnosis in British primary care. Patient Educ Couns. 1996;29:75-86.

7. Stewart M, Brown JB, Boon H, Galajda J, Meredith L, Sangster M. Evidence on patient-doctor communication. Cancer Prev Control. 1999;3:25-30.

8. Pyatak EA, Carandang K, Vigen CLP, Blanchard J, Diaz J, Concha-Chavez A, et al. Occupational therapy intervention improves glycemic control and quality of life among young adults with diabetes: the Resilient, Empowered, Active Living with Diabetes (REAL Diabetes) randomized controlled trial. Diabetes Care. 2018;41:696-704.

9. Svedbo Engström M, Leksell J, Johansson U-B, Borg S, Palaszewski B, Franzén S, et al. Health-related quality of life and glycaemic control among adults with type 1 and type 2 diabetes: a nationwide cross-sectional study. Health Qual Life Outcomes. 2019; 17:141.

10. Vandenberghe D, Albrecht J. The financial burden of non-communicable diseases in the European Union: a systematic review. Eur J Public Health. 2020;30:833-9.

11. Barceló A, Cafiero E, de Boer M, Mesa AE, Lopez MG, Jiménez RA, et al. Using collaborative learning to improve diabetes care and outcomes: the VIDA project. Prim Care Diabetes. 2010;4:145-53.

12. Epel ES, Crosswell AD, Mayer SE, Prather AA, Slavich GM, Puterman E, et al. More than a feeling: a unified view of stress measurement for population science. Front Neuroendocrinol. 2018;49:146-69.

13. Walker RJ, Garacci E, Campbell JA, Egede LE. The influence of daily stress on glycemic control and mortality in adults with diabetes. J Behav Med. 2020;43:723-31.

14. Bolaño E, Sarría A. Prespectiva de los pacientes sobre la diabetes tipo 2 y relación con los profesionales sanitarios de atención primaria. Rev Orig la Univ Alcalá. 2003;32:1-8.

15. Aromataris E, Fernandez R, Godfrey CM, Holly C, Khalil H, Tungpunkom P. Summarizing systematic reviews: methodological development, conduct and reporting of an umbrella review approach. Int J Evid Based Healthc. 2015;13:132-40. 
16. Tricco AC, Lillie E, Zarin W, O’Brien KK, Colquhoun H, Levac D, et al. PRISMA Extension for Scoping Reviews (PRISMA-ScR): checklist and explanation. Ann Intern Med. 2018;169:467-73.

17. Mokkink LB, Terwee CB, Patrick DL, Alonso J, Stratford PW, Knol DL, et al. The COSMIN checklist for assessing the methodological quality of studies on measurement properties of health status measurement instruments: an international Delphi study. Qual Life Res. 2010;19:539-49.

18. Toobert DJ, Hampson SE, Glasgow RE. The summary of diabetes self-care activities measure: results from 7 studies and a revised scale. Diabetes Care J. 2000;23:943-50.

19. Polonsky WH, Anderson BJ, Lohrer PA, Welch G, Jacobson AM, Aponte JE, et al. Assessment of diabetes-related distress. Diabetes Care. 1995;18:754-60.

20. Meadows K, Steen N, McColl E, Eccles M, Shiels C, Hewison $\mathrm{J}$, et al. The diabetes health profile (DHP): a new instrument for assessing the psychosocial profile of insulin requiring patients: development and psychometric evaluation. Qual Life Res. 1996;5:242-54.

21. Meadows KA, Abrams C, Sandbæk A. Adaptation of the Diabetes Health Profile (DHP-1) for use with patients with type 2 diabetes mellitus: psychometric evaluation and cross-cultural comparison. Diabet Med. 2000;17:572-80.

22. Schmitt A, Gahr A, Hermanns N, Kulzer B, Huber J, Haak T. The Diabetes Self-Management Questionnaire (DSMQ): development and evaluation of an instrument to assess diabetes selfcare activities associated with glycaemic control. Health Qual Life Outcomes. 2013;11:138.

23. Traina SB, Mathias SD, Colwell HH, Crosby RD, Abraham C. The Diabetes Intention, Attitude, and Behavior Questionnaire: evaluation of a brief questionnaire to measure physical activity, dietary control, maintenance of a healthy weight, and psychological antecedents. Patient Prefer Adherence. 2016;10:213-22.

24. Brod M, Hammer M, Christensen T, Lessard S, Bushnell DM. Understanding and assessing the impact of treatment in diabetes: The treatment-related impact measures for diabetes and devices (TRIM-Diabetes and TRIM-Diabetes Device). Health Qual Life Outcomes. 2009;7:83.

25. Naegeli AN, Hayes RP. Expectations about and experiences with insulin therapy contribute to diabetes treatment satisfaction in insulin-naïve patients with type 2 diabetes. Int J Clin Pract. 2010;64:908-16.

26. Hsu H-C, Chen S-Y, Huang Y-C, Wang R-H, Lee Y-J, An L-W, et al. Decisional balance for insulin injection: scale development and psychometric testing. J Nurs Res. 2019;27:e42.

27. Orozco-Beltrán D, Artola S, Jansà M, de la Torre-Casares ML, Fuster E. Impact of hypoglycemic episodes on health-related quality of life of type-2 diabetes mellitus patients: development and validation of a specific QoLHYPO@ questionnaire. Health Qual Life Outcomes. 2018;16:52.

28. Matza LS, Boye KS, Stewart KD, Paczkowski R, Jordan J, Murray LT. Development of the Diabetes Injection Device Experience Questionnaire (DID-EQ) and Diabetes Injection Device Preference Questionnaire (DID-PQ). J Patient Rep Outcomes. 2018;2:43.

29. Svedbo Engström M, Leksell J, Johansson UB, Eeg-Olofsson K, Borg S, Palaszewski B, et al. A disease-specific questionnaire for measuring patient-reported outcomes and experiences in the Swedish National Diabetes Register: development and evaluation of content validity, face validity, and test-retest reliability. Patient Educ Couns. 2018;101:139-46.

30. Schmitt A, Reimer A, Kulzer B, Icks A, Paust R, Roelver KM, et al. Measurement of psychological adjustment to diabetes with the diabetes acceptance scale. J Diabetes Complicat. 2018;32:384-92.
31. Alvarado-Martel D, Ruiz Fernández MA, Vigaray MC, Carrillo A, Boronat M, Montesdeoca AE, et al. ViDa1: the development and validation of a new questionnaire for measuring healthrelated quality of life in patients with type 1 diabetes. Front Psychol. 2017;8:1-14.

32. Mulhern B, Labeit A, Rowen D, Knowles E, Meadows K, Elliott $\mathrm{J}$, et al. Developing preference-based measures for diabetes: DHP-3D and DHP-5D. Diabet Med. 2017;34:1264-75.

33. Hilliard ME, Iturralde E, Weissberg-Benchell J, Hood KK. The Diabetes strengths and resilience measure for adolescents with type 1 diabetes (DSTAR-Teen): validation of a new, brief selfreport measure. J Pediatr Psychol. 2017;42:995-1005.

34. Traina SB, Colwell HH, Crosby RD, Mathias SD. Pragmatic measurement of health satisfaction in people with type 2 diabetes mellitus using the Current Health Satisfaction Questionnaire. Patient Relat Outcome Meas. 2015;6:103-15.

35. The Health and Social Care Information Centre. National diabetes audit 2013-14: patient experience of Diabetes Services Survey Pilot. 2014. https://files.digital.nhs.uk/publicationimpo rt/pub14xxx/pub14211/nda-peds-pil-13-14-rep.pdf. Accessed 17 Jan 2020.

36. Hayes RP, Schultz EM, Naegeli AN, Curtis BH. Test-retest, responsiveness, and minimal important change of the ability to perform physical activities of daily living questionnaire in individuals with type 2 diabetes and obesity. Diabetes Technol Ther. 2012;14:1118-25.

37. Arbuckle RA, Humphrey L, Vardeva K. Psychometric evaluation of the Diabetes Symptom Checklist-Revised (DSC-R): a measure of symptom distress. Value Health. 2009;12:1168-75.

38. Brod M, Skovlund SE, Wittrup-Jensen KU. Measuring the impact of diabetes through patient report of treatment satisfaction, productivity and symptom experience. Qual Life Res. 2006;15:481-91.

39. Anderson RT, Skovlund SE, Marrero D, Levine DW, Meadows K, Brod M, et al. Development and validation of the insulin treatment satisfaction questionnaire. Clin Ther. 2004;26:565-78.

40. Burroughs TE, Desikan R, Waterman BM, Gilin D, McGill J. Development and validation of the Diabetes Quality of Life Brief Clinical Inventory. Diabetes Spectr. 2004;17:41-9.

41. Anderson RM, Funnel MM, Fitzgerald JT, Marrero DG. The Diabetes Empowerment Scale. Diabetes Care. 2000;23:739-43.

42. Bradley C, Todd C, Gorton T, Symonds E, Martin A, Plowright $\mathrm{R}$. The development of an individualized questionnaire measure of perceived impact of diabetes on quality of life: the ADDQoL. Qual Life Res. 1999;8:79-91.

43. Talbot F, Nouwen A, Gingras J, Gosselin M, Audet J. The assessment of diabetes-related cognitive and social factors: the multidimensional diabetes questionnaire. J Behav Med. 1997;20:291-312.

44. Snoek FJ, Mollema ED, Heine RJ, Bouter LM, Van Der Ploeg HM. Development and validation of the diabetes fear of injecting and self- testing questionnaire (D-FISQ): first findings. Diabet Med. 1997;14:871-6.

45. Lewis KS, Bradley C, Knight G, Boulton AJM, Ward JD. A measure of treatment satisfaction designed specifically for people with insulin-dependent diabetes. Diabet Med. 1988;5:235-42.

46. Cox DJ, Irvine A, Gonder-Frederick L, Nowacek G, Butterfield J. Fear of hypoglycemia: quantification, validation, and utilization. Diabetes Care. 1987;10:617-21.

47. Testa MA, Simonson DC. Health economic benefits and quality of life during improved glycemic control in patients with type 2 diabetes mellitus. JAMA. 1998;280:1490-6.

48. Polonsky WH. Emotional and quality-of-life aspects of diabetes management. Curr Diab Rep. 2002;2:153-9.

49. Fisher L, Mullan JT, Arean P, Glasgow RE, Hessler D, Masharani U. Diabetes distress but not clinical depression or depressive 
symptoms is associated with glycemic control in both cross-sectional and longitudinal analyses. Diabetes Care. 2010;33:23-8.

50. Lim SM, Siaw MYL, Tsou KYK, Kng KK, Lee JY-C. Risk factors and quality of life of patients with high diabetes-related distress in primary care: a cross-sectional, multicenter study. Qual Life Res. 2019;28:491-501.

51. Strandberg RB, Graue M, Wentzel-Larsen T, Peyrot M, Rokne B. Relationships of diabetes-specific emotional distress, depression, anxiety, and overall well-being with HbA1c in adult persons with type 1 diabetes. J Psychosom Res. 2014;77:174-9.

52. Van Bastelaar KMP, Pouwer F, Geelhoed-Duijvestijn PHLM, Tack CJ, Bazelmans E, Beekman AT, et al. Diabetes-specific emotional distress mediates the association between depressive symptoms and glycaemic control in type 1 and type 2 diabetes. Diabet Med. 2010;27:798-803.

53. Young-Hyman D, de Groot M, Hill-Briggs F, Gonzalez JS, Hood K, Peyrot M. Psychosocial care for people with diabetes: a position statement of the American Diabetes Association. Diabetes Care. 2016;39:2126-40.

54. Lau CY, Qureshi AK, Scott SG. Association between glycaemic control and quality of life in diabetes mellitus. J Postgrad Med. 2004;50:189-93 (Discussion 194)

55. Ishii $\mathrm{H}$, Niiya $\mathrm{T}$, Ono $\mathrm{Y}$, Inaba $\mathrm{N}$, Jinnouchi $\mathrm{H}$, Watada $\mathrm{H}$. Improvement of quality of life through glycemic control by liraglutide, a GLP-1 analog, in insulin-naive patients with type 2 diabetes mellitus: the PAGE1 study. Diabetol Metab Syndr. 2017;9:3.

56. El Achhab Y, Nejjari C, Chikri M, Lyoussi B. Disease-specific health-related quality of life instruments among adults diabetic: a systematic review. Diabetes Res Clin Pract. 2008;80:171-84.

57. Norris SL, McNally TK, Zhang X, Burda B, Chan B, Chowdhury FM, et al. Published norms underestimate the health-related quality of life among persons with type 2 diabetes. J Clin Epidemiol. 2011;64:358-65.

58. Fisher L, Tang T, Polonsky W. Assessing quality of life in diabetes: I. A practical guide to selecting the best instruments and using them wisely. Diabetes Res Clin Pract. 2017;126:278-85.

59. Mira JJ, Nuño-Solinís R, Guilabert-Mora M, Solas-Gaspar O, Fernández-Cano P, González-Mestre MA, et al. Development and validation of an instrument for assessing patient experience of chronic illness care. Int J Integr Care. 2016;16:13.

60. Briggs MS, Rethman KK, Crookes J, Cheek F, Pottkotter K, McGrath S, et al. Implementing patient-reported outcome measures in outpatient rehabilitation settings: a systematic review of facilitators and barriers using the consolidated framework for implementation research. Arch Phys Med Rehabil. 2020;101:1796-812.

61. Foster A, Croot L, Brazier J, Harris J, O'Cathain A. The facilitators and barriers to implementing patient reported outcome measures in organisations delivering health related services: a systematic review of reviews. J Patient Rep Outcomes. 2018;2:46.

62. Engström MS, Leksell J, Johansson U-B, Gudbjörnsdottir S. What is important for you? A qualitative interview study of living with diabetes and experiences of diabetes care to establish a basis for a tailored patient-reported outcome measure for the Swedish National Diabetes Register. BMJ Open. 2016;6:e010249. https:// doi.org/10.1136/bmjopen-2015-010249

63. Wilkinson A, Whitehead L, Ritchie L. Factors influencing the ability to self-manage diabetes for adults living with type 1 or 2 diabetes. Int J Nurs Stud. 2014;51:111-22.

64. Berwick DM, Nolan TW, Whittington J. The triple aim: care, health, and cost. Health Aff. 2008;27:759-69.

65. Wagner EH, Austin BT, Von Korff M. Organizing care for patients with chronic illness. Milbank Q. 1996;74:511-44.

66. Coleman K, Austin BT, Brach C, Wagner EH. Evidence on the chronic care model in the new millennium. Health Aff. 2009;28:75-85.

67. Borg S, Eeg-Olofsson K, Palaszewski B, Engström MS, Gerdtham U-G, Gudbjörnsdottir S. Patient-reported outcome and experience measures for diabetes: development of scale models, differences between patient groups and relationships with cardiovascular and diabetes complication risk factors, in a combined registry and survey study in Sweden. BMJ Open. 2019;9:e025033.

68. Bradley C. Handbook of psychology and diabetes. 1st ed. London: Routledge; 1994.

69. Mills A. Health care systems in low- and middle-income countries. N Engl J Med. 2014;370:552-7. 